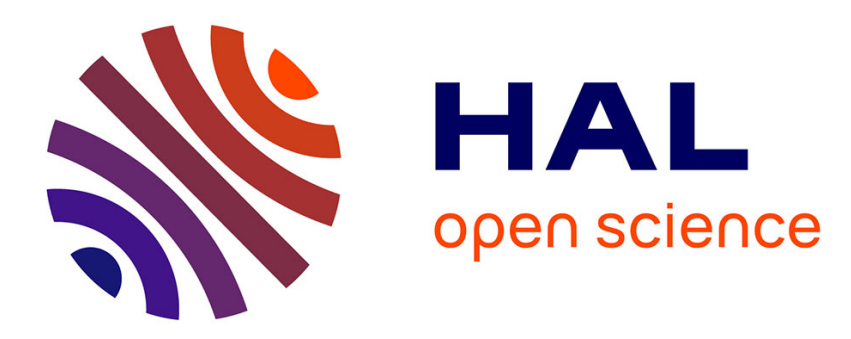

\title{
Les méthodes de déconvolution et leurs limitations fondamentales
}

\author{
Y.G. Biraud
}

\section{To cite this version:}

Y.G. Biraud. Les méthodes de déconvolution et leurs limitations fondamentales. Revue de Physique Appliquée, 1976, 11 (2), pp.203-214. 10.1051/rphysap:01976001102020300 . jpa-00244050

\section{HAL Id: jpa-00244050 https://hal.science/jpa-00244050}

Submitted on 1 Jan 1976

HAL is a multi-disciplinary open access archive for the deposit and dissemination of scientific research documents, whether they are published or not. The documents may come from teaching and research institutions in France or abroad, or from public or private research centers.
L'archive ouverte pluridisciplinaire HAL, est destinée au dépôt et à la diffusion de documents scientifiques de niveau recherche, publiés ou non, émanant des établissements d'enseignement et de recherche français ou étrangers, des laboratoires publics ou privés. 


\title{
LES MÉTHODES DE DÉCONVOLUTION ET LEURS LIMITATIONS FONDAMENTALES
}

\author{
Y. G. BIRAUD \\ Laboratoire : Groupe Infra-Rouge Spatial de 92190 Meudon, France
}

\begin{abstract}
Résumé. - Tout d'abord, nous rappelons les analogies entre systèmes physiques linéaires et équations de convolution.

Nous montrerons ensuite les difficultés théoriques rencontrées pour résoudre ces équations (existence, unicité des solutions...).

Puis nous définirons, précisément, les termes déconvolution, désapodisation au sens des physiciens.

Nous aborderons enfin une revue, non exhaustive, des méthodes et algorithmes de déconvolution couramment proposés par différents auteurs.

Nous montrerons quand cela est possible les limitations amenées par les bruits apparaissant aux différents stades de la mesure et comment dans certains cas une information a priori peut réduire ces limitations.
\end{abstract}

\begin{abstract}
We deal first with the analogy between physical linear systems and convolution equations, and show the theoretical difficulties encountered when solving such equations (existence, uniqueness...).

We define precisely the words deconvolution, apodization used by physicists.

We then make a survey (not intended to be exhaustive) of deconvolution methods and algorithms currently proposed by severals authors.

In the end we study, when possible, the limitations due to various noises appearing at different levels of the measurement and how, in some circumstances, an a priori information may decrease the importance of these limitations.
\end{abstract}

\section{DÉCONVOLUTION}

1. Essai de définition du mot. - Pour les mots convolution ou composition tout le monde est d'accord sur la définition suivante :

$$
h(x)=\int_{-\infty}^{+\infty} f(t) g(x-t) \mathrm{d} t
$$

ou

$$
h(x)=f(x) * g(x)
$$

$h(x)$ est le résultat de la convolution de la fonction $f$ par la fonction $g$. Supposons les fonctions $h$ et $f$ connues, alors l'éq. (1) est dite équation de convolution. C'est une équation de Fredholm de $2^{\mathrm{e}}$ espèce dont le noyau $g$ ne dépend que de la différence $x-t$. Notons aussi les rapports étroits entre la convolution notée $*$ et la corrélation.

On peut proposer la définition suivante du mot déconvolution: c'est la résolution d'une équation de convolution. Cette définition nous parait la seule possible.

Nous déplorons, personnellement qu'il n'existe à notre connaissance de définition officielle du mot. Chacun utilise le mot déconvolution dans un sens

Astronomie Infrarouge, Equipe de Recherche Associée au C. N. R. S., $\mathbf{n}^{\circ} 377$.

REVUE DE PHYSiQue APPLIQUÉE. - T. 11, No 2, MARS 1976 différent et dans bien des cas cela amène à de grosses confusions.

Nous pensons que pour un cas physique bien déterminé on doit préciser. Par exemple, nous appelerons déconvolution avec bruit de mesure la résolution de l'équation :

où

$$
\begin{aligned}
& k(x)=f(x) * g(x) \\
& k(x)=h(x)+n_{2}(x)
\end{aligned}
$$

$h(x)$ étant une fonction sûre,

$n_{2}(x)$ étant une réalisation d'une fonction aléatoire.

Nous définirons au cours de l'exposé les notions de :

- déconvolution avec ou sans contrainte,

- déconvolution avec ou sans élargissement de bande,

- déconvolution statistique.

2. La convolution en physique expérimentale. - Les mesures physiques sont dans la grande majorité des cas réalisées à l'aide de systèmes linéaires (ou parfois on utilise la partie linéaire de la caractéristique). C'est pourquoi on trouve si souvent et dans des domaines très variés la notion de convolution. En effet, l'on sait que la condition nécessaire et suffisante pour qu'un système physique $\mathcal{A}$ soit défini par un opérateur de convolution est que ce système soit :

- linéaire (au sens mathématique du terme), 
- continu

— invariant par translation.

Cette condition, très bien perçue intuitivement, fait appel à des développements mathématiques dans lesquels nous n'entrerons pas. Nous nous contenterons de la traduire.

Si $e(x)$ est l'entrée (la quantité à mesurer) du système $\mathcal{A}$ et $s(x)$ est sa sortie (la mesure de $e(x)$ ), alors $s$ est liée à $e$ par la relation :

$$
s(x)=e(x) * A(x)
$$

où $s, e$ et $A$ sont des fonctions ou des distributions.

La signification de $A(x)$ est simple. Si $e(x)=\delta(x),(2)$ devient

$$
A(x)=\delta(x) * A(x)
$$

car la distribution de Dirac est l'unité de convolution. Donc $A(x)$ caractérise parfaitement le système linéaire : c'est la réponse impulsionnelle. Remarquons dès maintenant que, dans les cas où $A(x)$ n'est pas connu théoriquement mais est elle-même le résultat d'une mesure, on n'a pas accès à $A(x)$ exactement mais à une approximation. C'est par exemple le cas en Optique ou en Mécanique des vibrations.

La situation, en Physique expérimentale, peut être encore compliquée et peut se résumer sur le schéma suivant :

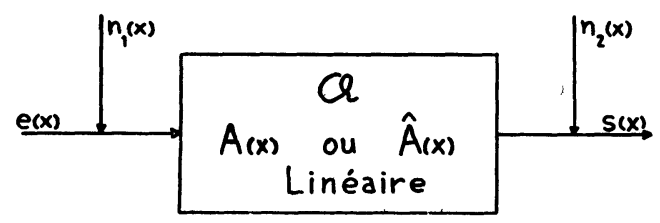

Fig. 1.

La grandeur d'entrée $e(x)$ que l'on veut estimer est très souvent affectée d'un bruit $n_{1}(x)$. C'est le cas si un canal fluctuant s'interpose entre $e(x)$ et le système physique $\mathcal{A}$ (atmosphère fluctuante en Astronomie par exemple).

On ne connaît pas exactement $A(x)$ mais une estimée $\widehat{A}(x)$ qui comme nous l'avons dit peut être une mesure bruitée et distordue de $A(x)$.

Enfin, la sortie $s(x)$ est toujours bruitée par le bruit propre du système de détection.

Les bruits que nous venons d'évoquer peuvent être additifs ou multiplicatifs et de statistiques connues ou inconnues. On doit tenir compte de leurs propriétés avant d'élaborer une méthode de déconvolution.

Notons dès maintenant l'importance de ces bruits. Ils constituent le plus souvent, la limitation drastique de bien des méthodes proposées dans la littérature.

3. Déconvolution. Point de vue théorique. - Nous nous restreindrons aux seuls cas (physiques) des fonctions ou distributions tempérées ayant une TF. Nous avons donc à résoudre :

$$
s(x)=e(x) * A(x)
$$

ou son équivalent

$$
S(v)=E(v) * a(v)
$$

avec

$$
\begin{aligned}
& S(v) \rightleftharpoons s(x) \\
& E(v) \stackrel{\mathrm{TF}}{\rightleftharpoons} e(x) \\
& a(v) \rightleftharpoons A(x) .
\end{aligned}
$$

où TF est mis pour Transformée de Fourier.

Nous tenons ici à insister sur l'importance de la TF. En effet, on sait que les exponentielles complexes sont fonctions propres des filtres linéaires. Dans le cas où $a(v)$ est indéfiniment dérivable (c'est très souvent le cas en Physique) on peut montrer [1] (Biraud, Y. G.) que ce sont les seules.

On voit donc l'intérêt que l'on a à décomposer entrée et sortie sur une base complète orthonormale qui se conserve lors du passage à travers le filtre. Ceci n'empêche pas de choisir toute autre base analogue (Walsh-Hadamard par exemple) pour la mise en œuvre d'un algorithme rapide. Ceci est recommandé dans les méthodes comportant un va et vient entre l'espace direct et l'espace transformé.

Le problème se pose de l'existence de solutions à l'éq. (2).

Plaçons nous dans une algèbre de convolution $\mathcal{B}$ (espace vectoriel de distribution $-\delta$ inclus - sur lequel on peut définir le produit de convolution d'un nombre fini de distributions). Alors pour que (2) ait toujours au moins une solution dans $\mathcal{B}$ et $\forall s \in \mathcal{B}$ il faut et il suffit que $A(x)$ possède un inverse de convolution $A^{*-1}$ tel que

$$
A * A^{*-1}=\delta
$$

alors la résolution de (2) est immédiate.

$$
\begin{aligned}
s(x) * A^{*-1}(x)=e(x) * A(x) * A^{*-1}(x) & = \\
& =e(x) * \delta(x)=e(x) .
\end{aligned}
$$

Donc

$$
e(x)=A^{*-1}(x) * s(x) .
$$

Mais $A^{*-1}(x)$ n'existe pas toujours. Par exemple, si $A$ est une distribution. De façon plus grave $A^{*-1}$ ne peut exister que pour certaines fonctions $s(x)$. Notons dès maintenant le point suivant, si l'algèbre $\mathfrak{B}$ admet des diviseurs de zéro alors l'éq. (2) admet une infinité de solutions.

L'éq. (3) peut s'écrire

$$
E(v)=\frac{1}{a(v)} \cdot S(v) .
$$

Mais il ne faut pas perdre de vue l'aspect formel de 
cette solution [2] (Arsac, J.). Il faut que $1 / a(v)$ existe et soit une distribution tempérée. Si $a(v)$ est une fonction qui ne s'annule pour aucune valeur de $v$ et qui ne tende pas vers zéro à l'infini plus vite qu'une puissance de $1 / v$ alors on peut définir $1 / a(v)$ de façon unique.

Nous voyons que ces conditions sont sévères. Dans la pratique elles ne sont jamais réalisées. En Optique et Radioastronomie par exemple $a(v)$ est une fonction qui théoriquement s'annule au-delà d'une fréquence de cut-off $v_{\text {co. }}$. Mais dans la majorité des cas pratiques $|a(v)| \rightarrow 0$ quand $v$ croît. Même dans ce cas, $a(v)$ étant le plus souvent affecté d'un bruit de mesure $n_{a}$ il existera une certaine fréquence $v_{0}$ au-delà de laquelle on aura

$$
|a(v)| \sim \sigma_{n_{a}}
$$

$\sigma_{n_{a}}$ étant l'e. q. m. du bruit $n_{a}$.

Alors $a(v)$ présentera de multiples passages par zéro et la division $1 / a(v)$ sera impossible.

Pour ce qui est de l'unicité des solutions elle n'est jamais démontrable dans les cas pratiques. Considérons le cas des grandeurs non bruitées et supposons que :

$$
|a(v)| \equiv 0 \quad \forall|v|>v_{\text {co }}
$$

alors l'équation

$$
S(v)=E(v) \cdot a(v)
$$

nous montre que

$$
S(v) \equiv 0 \quad \forall|v|>v_{\mathrm{co}}
$$

et ce quelle que soit la forme de $E(v)$ au-delà de $v_{\text {co }}$.

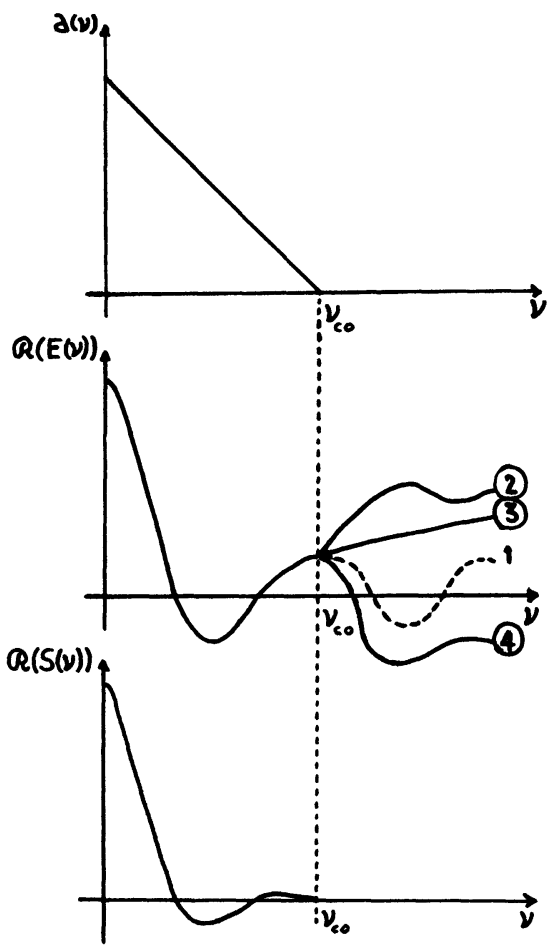

FIG. 2.
Toutes les fonctions $\widehat{E}_{\mathbf{j}}(v)$ et $\widehat{E}_{\mathbf{j}}(v)$ telles que

$$
\begin{array}{ll}
\widehat{E}_{\mathrm{i}}(v)=\widehat{E}_{\mathrm{j}}(v) & \forall v \in\left[-v_{\mathrm{co}}, v_{\mathrm{co}}\right] \\
\widehat{E}_{\mathrm{i}}(v) \neq \widehat{E}_{\mathrm{j}}(v) & \forall v \notin\left[-v_{\mathrm{co}}, v_{\mathrm{co}}\right]
\end{array}
$$

seront filtrées de la même manière par $\mathcal{A}$. Les prolongements (2), (3) ou (4) de la figure 2 sont tous possibles. Les restitutions $\hat{e}_{2}(x), \hat{e}_{3}(x)$ et $\hat{e}_{4}(x)$ sont des entrées qui donneront la même sortie $s(x)$. L'ensemble infini des fonctions $\widehat{E}_{\mathrm{i}}(v)$ définies plus haut forme ce que l'on appelle souvent les solutions muettes $\varepsilon_{\mathrm{m}}$.

Il est extrêmement rare, à notre avis, que l'expérimentateur puisse choisir parmi ces solutions. Par contre, s'il dispose d'informations, il pourra trouver dans $\varepsilon_{\mathrm{m}}$ un sous ensemble $G_{\mathrm{i}}$ et il forcera alors sa solution à appartenir à $G_{1}$.

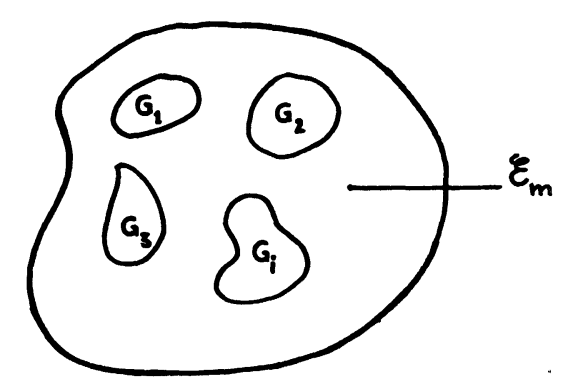

Fig. 3.

Nous ne connaissons pas, pour notre part, de cas où $G_{\mathrm{i}}$ soit réduit à un point. Donc, le maximum que l'on puisse espérer c'est de trouver une famille de fonctions $\widehat{E}_{\mathrm{i}}(v)$ ou $\hat{e}_{\mathrm{i}}(x)$ qui fourniront une sortie $s_{\mathrm{i}}(x)$ donc seront en accord avec la mesure faite et vérifieront un certain critère choisi.

4. Influence de l'échantillonnage et de la quantification. - Nous nous intéresserons ici au problème de la déconvolution numérique (sans ignorer pour autant les méthodes analogiques opérant tant en Electronique qu'en Optique).

Le signal $s(x)$ n'est, pour des raisons évidentes, mesuré que sur un intervalle de mesure finie (temps d'expérience fini). Pour $e(x)$ deux cas se présentent :

- $e(x)$ est, par nature, défini sur un support borné (Optique, Astronomie optique ou radio).

- $e(x)$ n'est pas à support borné. Il est évident, dans ce cas, que nous sommes contraints de restreindre le support de $e(x)$.

Le signal $A(x)$, lui, est le plus souvent de support infini (cas de l'Electronique, de l'Optique et de l'Astronomie).

Dans tous les cas où $s(x)$ et $A(x)$ sont à support infini, nous sommes contraints de ne numériser ces fonctions que sur un support d'extension finie soit $[-X, X]$. Ce faisant, nous commettons nécessairement une erreur. 
Rappelons en effet une propriété des transformées de Fourier des fonctions définies sur un support borné. $\mathrm{Si}$

si

$$
f(x) \rightleftharpoons F(v)
$$

alors

$$
f(x) \equiv 0 \quad \forall x \notin[-X, X]
$$

$F(v)$ est une fonction analytique

Or, une fonction analytique ne peut être nulle sur un support, si petit soit-il, sans être identiquement nulle partout. Donc

$$
F(v) \text { définie sur }]-\infty,+\infty[\text {. }
$$

Le théorème d'échantillonnage (dit souvent de Shannon/Nyquist mais démontré par Cauchy) peut donc s'appliquer à $F(v)$ : la connaissance des échantillons $F_{\mathrm{p}}=F(p \Delta v)$

$$
(p=-\infty, \ldots,-2,-1,0,1,2, \ldots,+\infty)
$$

permet de reconstituer $F(v)$ en tout point par la formule :

$$
F(v)=\sum_{p=-\infty}^{+\infty} F(p \Delta v) \cdot \frac{\sin \pi / \Delta v(v-p \Delta v)}{\pi / \Delta v(v-p \Delta v)}
$$

à la condition que

$$
\Delta v \leqslant \frac{1}{2 X} .
$$

Si maintenant, nous désirons échantillonner $f(x)$ et pouvoir la reconstituer identiquement par la suite, nous voyons que nous sommes théoriquement obligés de l'échantillonner avec un pas infiniment fin puisque sa TF $F(v)$ est définie sur ] $-\infty,+\infty[$.

Ceci nous montre donc que, stricto sensu, l'on ne peut échantillonner $f(x)$. Dans les cas physiques courants on peut souvent admettre que l'énergie de $F(v)$ en dehors de la bande [ $\left.-v_{\mathrm{sh}}, v_{\mathrm{sh}}\right]$ est une fraction faible de celle contenue dans la même bande. Néanmoins et en toute rigueur [3] (Standish, C. J.) on ne peut le faire.

L'erreur commise à la reconstruction peut être soit localement infinie, soit d'intégrale infinie et ce dans des cas non pathologiques. En fait, Standish s'est intéressé à des trains d'impulsions périodiques.

Rappelons que si l'on désire calculer la TF d'un signal $s(x)$ de bande $\left[-v_{s}, v_{s}\right]$ et noyé dans un bruit de bande [ $\left.-v_{\mathrm{n}^{\prime}}, v_{\mathrm{n}}\right]$ où $v_{\mathrm{n}}>v_{\mathrm{s}}$ (ou a fortiori blanc) il est impératif d'échantillonner $s(x)$ à la cadence

$$
h \leqslant \frac{1}{2 v_{\mathrm{n}}} \text {. }
$$

Si ceci n'est pas réalisé, un phénomène de repli des spectres de bruit se produit et le spectre du signal (et du bruit) sur la bande $\left[-v_{s}, v_{s}\right]$ est irrémédiablement déformé. En d'autres termes, les opérations de filtrage et d'échantillonnage ne commutent pas. Un moyen pratique pour savoir si l'échantillonnage de $s(x)+n(x)$ est correct est de le suréchantillonner le plus possible soit $\mathrm{h}$ la période d'échantillonnage - et de s'assurer que la TF de $s(x)+n(x)$ est nulle et ne présente pas de discontinuité en $v_{\max }=1 /(2 h)$.

Cette condition est nécessaire sans être suffisante. Si $S(v)+N(v)$ s'annule en $v_{0} \leqslant v_{\max }$ il suffira d'échantillonner avec une période

$$
h_{0}=\frac{1}{2 v_{0}} .
$$

Nous rencontrerons dans la suite des méthodes où le pas d'échantillonnage influe sur la qualité de la restitution.

Signalons enfin les effets de quantification. Peu de travaux à notre connaissance ont été publiés sur les erreurs de reconstitution d'un signal à partir d'échantillons quantifiés (Vinokur, M.) [4].

Il semble que pour un pas de quantification $q$ compris entre 0,1 et $1 \sigma(\sigma:$ e. q. m. du bruit) les erreurs de quantification soient inférieures à celles dues au bruit lui-même.

Rappelons enfin (Biraud, Y. G.) [5] que dans le calcul de toute TF la précision requise pour le calcul des lignes trigonométriques doit être suffisante. Une précision de 8 bits amène des erreurs de spectre non blanc et qui peuvent dépasser $10^{-2}$.

5. Revue de quelques méthodes de déconvolution, sans extension de bande. - Dans ce paragraphe nous ne tenterons pas une revue exhaustive des différentes méthodes utilisées. Nous tenterons d'analyser les plus répandues ou celles qui semblent dictées par le bon sens. On trouvera par ailleurs [6], [7], [8], des études assez complètes de différentes méthodes. Nous essaierons ici d'analyser les avantages et les inconvénients de certaines d'entre elles et tenterons de faire ressortir les idées générales que l'on doit avoir à l'esprit avant l'étude ou l'application de tout processus de déconvolution :

5.1 INVERSION DIRECTE DE LA MATRICE DE CONVOLUTION. - L'équation intégrale de convolution :

$$
s(x)=A(x) * e(x)+n(x)
$$

peut se transformer en une équation matricielle. Notons tout de suite ici l'importance du choix du pas d'échantillonnage $h$. On doit tout d'abord filtrer - si ce n'est déjà fait - $s(x)$ afin d'éliminer les composantes de bruit qui ne seraient pas dans la bande signal. Ceci fait, le pas de Shannon h suffit pour échantillonner $s(x)$ et $A(x)$. Alors, l'équation (5) peut s'écrire sous forme matricielle :

$$
\mathcal{S}=\mathcal{A} \varepsilon+\mathcal{N}
$$

$\mathcal{A}$ peut être ramenée à une matrice carrée qui ne comporte comme coefficients que des valeurs $A_{n}=A(n \mathrm{~h})$ de la fonction d'appareil.

Supposons $A(x)$ connue sans bruit. Alors si $\mathcal{A}$ est 
inversible ([9] (Escudié, B.)), il suffit de multiplier la relation (6) à gauche par $\mathcal{A}^{-1}$

ou

$$
\mathcal{A}^{-1} S=A^{-1} \mathcal{A} E+A^{-1} \mathcal{N}
$$

$$
\mathcal{E}=\mathcal{A}^{-1} S-\mathcal{A}^{-1} \mathcal{N}
$$

Le problème principal qui se pose dans cette méthode est l'inversion brutale de la matrice $\mathfrak{A}$. La présence de bruits insignifiants sur $A(x)$ (signal/bruit de 2000 !) donne des résultats très mauvais (voir Fig. 4) [10] (Phillips, D. L.) et conduit à des oscillations de grandes amplitudes. Ceci tient à la nature de la matrice $\mathcal{A}$ (matrice grille) qui ne comporte de termes non nuls qu'au voisinage de la diagonale, ce qui fait que $A^{-1}$ est formée d'éléments de module important. Ce qui rend le terme $A^{-1} \mathcal{N}$ important.

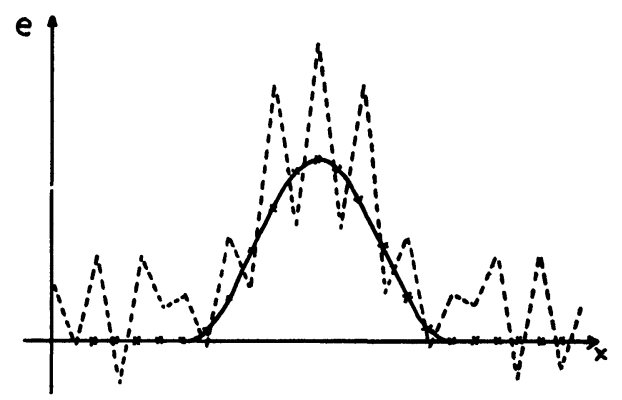

FIG. 4. - D'après Phillips. L'entrée $e(x)$. - - La restitution est affectée d'oscillations inhérentes aux méthodes d'inversion de matrice.

D'autre part, on peut expliquer l'apparition des oscillations par le fait que si l'on a suréchantillonné (cela revient à essayer d'étendre la bande au-delà de la fréquence $v_{\text {co}}$ ), toute sinusoïde de fréquence supérieure à $v_{\text {co }}$ et rajoutée à $\delta$ ne viole pas l'éq. (6) et sera donc présente dans la solution finale.

Signalons une méthode itérative de résolution de (6) dans ce sens faite par B. Escudié dans le cadre des conditions assurant la convergence du procédé et qui a obtenu de bons résultats (voir Fig. 5).

La méthode a été améliorée [10] (Phillips, D. L.). Pour éviter les oscillations on peut minimiser les différences secondes de la restitution en même temps que les carrés des différences entre la sortie et la restitution filtrée par $A(x)$ soit

où

$$
\alpha \sum_{n}\left(\hat{e}_{n+1}-2 \hat{e}+\hat{e}_{n-1}\right)^{2}+\sum_{n} d_{n}^{2}
$$

$\min$

$\widehat{e_{n}}=\widehat{e}(n \mathrm{~h})$ est le $n$-ième échantillon de restitution et

$$
d_{k}=s_{k}-\sum_{n} e_{n} A_{k-n}
$$

le problème est de fixer le coefficient $\alpha$ qui assure la régularité aux dépens de l'approximation de l'entrée. $\alpha$ est déterminé par essais successifs.

On peut généraliser cette approche et montrer [11]

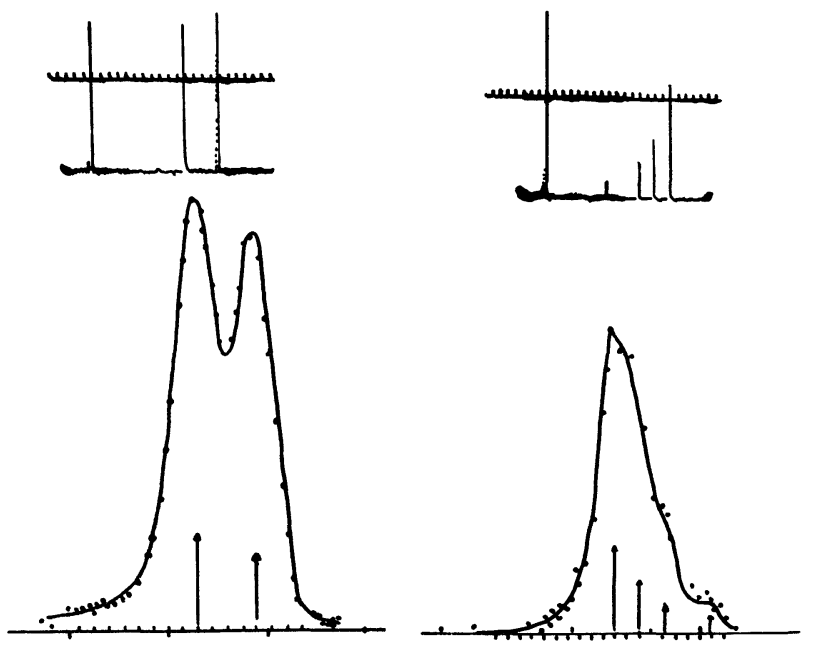

Fig. 5. - D'après Escudié. Deux exemples en spectroscopie nucléaire. En traits pleins : les mesures $s(x)$. Les flèches indiquent les positions et amplitudes théoriques des raies. En haut : les restitutions (sorties d'oscilloscope pour lesquelles le temps se déroule de droite à gauche). La raie à l'origine ne sert qu'à tester le bon fonctionnement de l'appareil.

(Twomey, S.) que l'on peut minimiser toute quantité quadratique en $\hat{e}$, qu'on peut inclure la minimisation de la distance de $\hat{e}$ à une courbe fixée $m$

$$
\sum_{n}\left(\hat{e}_{n}-m_{n}\right)^{2}
$$

ou qu'on peut minimiser une combinaison linéaire de ces deux quantités. Dans cette méthode, l'avantage est que la matrice $A$ n'est plus nécessairement carrée, donc que l'on peut traiter des problèmes sur ou sous contraints [12] (Tournarie, M).

5.2 MÉTHODES DITES DE « MODEL-FITTING » [13] (Dolby, R. M.). - Quand l'expérimentateur dispose d'informations précises sur $e(x)$ il peut être tenté de modéliser $e(x)$. Par exemple en Spectroscopie où la forme des raies est souvent connue. On peut aussi avoir une idée a priori sur le nombre et la position de ces raies. Prenons à titre d'exemple un modèle consistant en 3 raies supposées Lorentziennes centrées en $x_{1}, x_{2}, x_{3}$ d'amplitudes $a_{1}, a_{2}, a_{3}$ et de $\frac{1}{2}$ largeurs à mi-hauteur $\sigma_{1}, \sigma_{2}, \sigma_{3}$. La convolution de ce modèle $m(x)$ par la fonction instrumentale, $A(x)$ donne alors $s_{m}(x)$

$$
s_{m}(x)=A(x) * m(x) .
$$

On calcule alors une distance, par exemple quadratique, entre la mesure vraie $s(x)$ et $s_{m}(x)$

$$
\sum\left|s(x)-s_{m}(x)\right|^{2}=Q(\mathbf{a}, \mathbf{x}, \boldsymbol{\sigma})
$$

quantité qui dépend des paramètres du modèle. La minimisation de $Q$ fournit les valeurs des différents paramètres.

Les inconvénients de cette méthode sont multiples :

- il est difficile, voire impossible, de calculer les erreurs sur $\mathbf{a}, \mathbf{x}$ et $\boldsymbol{\sigma}$, 
- il est difficile d'estimer la sensibilité du modèle aux différents paramètres,

- enfin, cette méthode est souvent négative en ce sens que $Q_{\min }$, bien qu'atteint, est très supérieur à la puissance de bruit sur l'image. Ceci signifie que le modèle essayé est impossible. Il faut alors en inventer d'autres jusqu'à obtenir une solution acceptable. Mais quelle est la validité de celle-ci ? Elle n'est sûrement pas unique surtout en présence d'un bruit important. De plus, la forme du signal de sortie conduit souvent à construire de faux modèles (voir Fig. 6).

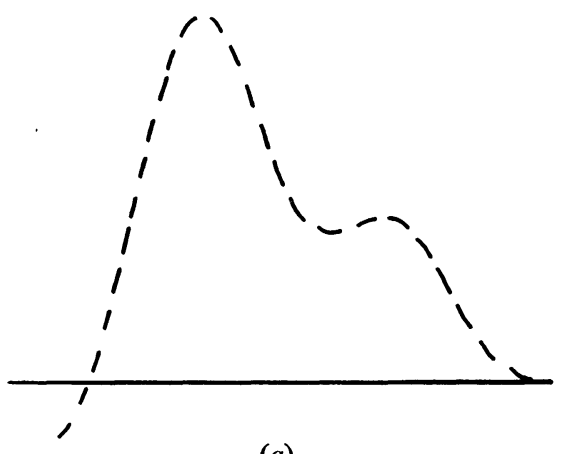

(a)

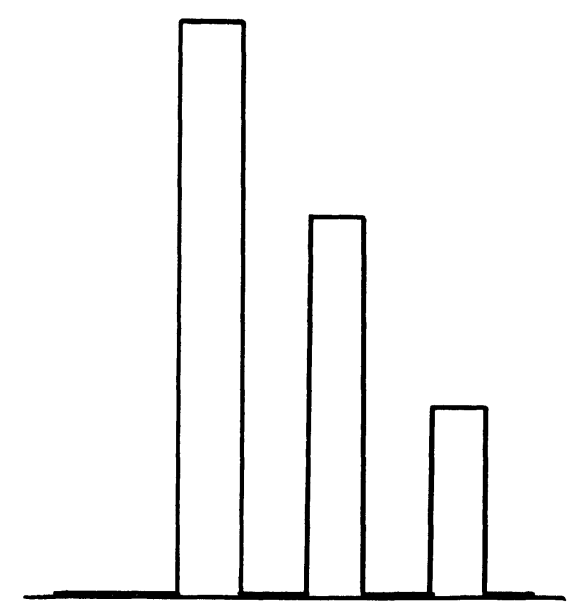

(b)

Fig. 6. - a) Le résultat de la convolution par un $\sin (k x) / k x$ de b) L'entrée inconnue. Le résultat $a$ ) n'incite pas à construire un modèle à 3 composantes tel $b$ ).

Il est par exemple évident qu'un petit satellite de faible contraste dans l'aile d'une raie ne peut être trouvé ainsi. Sa localisation et son amplitude ne peuvent être identifiées qu'à l'aide d'informations a priori et avec un signal de sortie à très grand rapport signal sur bruit.

5.3 LES MÉTHODES LINÉAIRES. - Nous les opposons aux méthodes non linéaires qui tendent à extrapoler la bande passante spatiale.

Partons de l'équation :

$$
s(x)=A(x) * e(x)+n(x)
$$

ou de sa TF

$$
S(v)=a(v) E(v)+N(v) .
$$

Nous appelons linéaire toute méthode qui applique un filtre linéaire à la sortie $s(x)$ afin d'obtenir une estimation de l'entrée $e(x)$. C'est donc une méthode qui peut s'appliquer :

- soit dans l'espace direct. Alors

$$
\hat{e}(x)=f(x) * s(x)
$$

où $f(x)$ est la réponse impulsionnelle du filtre ;

- soit dans l'espace des fréquences

$$
\widehat{E}(v)=F(v) . S(v)
$$

avec

$$
F(v) \rightleftharpoons f(x) .
$$

5.3.1 Le filtrage inverse. - Comme on l'a déjà vu (8) peut s'écrire formellement :

$$
E(v)-\frac{S(v)}{a(v)}-\frac{N(v)}{a(v)} \quad \forall v \text { t.q. } a(v) \neq 0
$$

et dans le cas non bruité

$$
E(v)=\frac{S(v)}{a(v)} \text { avec } a(v) \neq 0
$$

Nous appellerons cette méthode filtrage inverse ou compensation des effets instrumentaux en rajoutant sans extension de bande passante.

Ce filtrage s'il est effectué dans l'espace des fréquences (c'est l'entrée de beaucoup de méthodes dont par exemple celle de Biraud) conduit à ce que nous appellerons dans la suite Solution Principale au sens de Bracewell.

Notons que la division (11) devient strictement impossible quand

$$
a(v) \sim \sigma_{N}
$$

$\sigma_{N}$ e. q. m. de l'échantillon de bruit.

Si $v_{\text {co }}$ est la fréquence de cut-off due à l'instrument alors la division (11) doit se réaliser sur l'intervalle

$$
\left[-v_{\text {utile }}, v_{\text {utile }}\right] \text { avec } v_{\text {utile }} \ll v_{\text {co }} \text {. }
$$
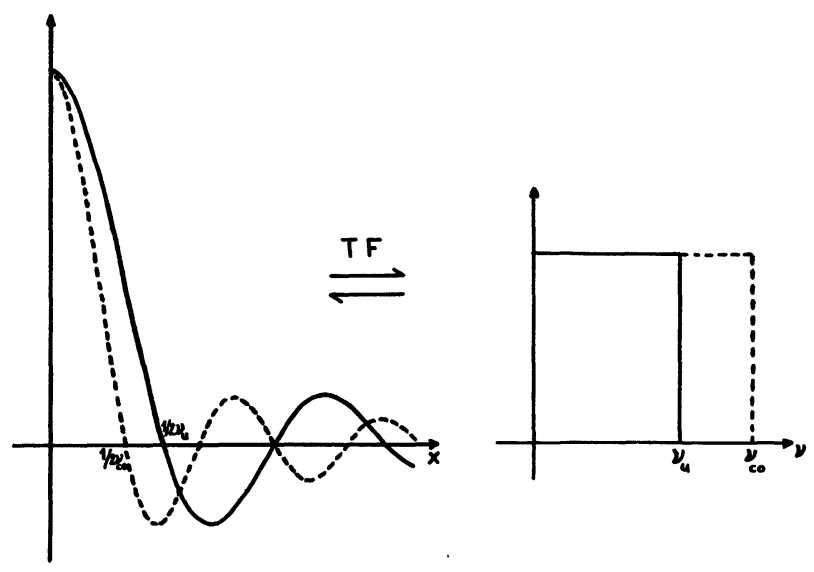

Fig. 7. - Illustration de la perte en pouvoir de résolution. a) - - L La fonction instrumentale correspondant au filtre inverse parfait de bande $v_{\text {co }}$. Ce qu'elle devient si l'on restreint la bande à $v_{u}$. Noter les importants pieds négatifs qui justifient les techniques d'apodisation. 
Mais ceci est un inconvénient important car on rétrécit encore la bande passante et la résolution qui était $1 /\left(2 v_{\text {co }}\right)$ devient $1 /\left(2 v_{\text {utile }}\right)$.

De plus, ce filtrage revient à convoler l'entrée vraie $e(x)$ par

$$
\frac{\sin k \pi x}{k \pi x}
$$

qui est très oscillant et présente des pieds négatifs importants $(20 \%)$. D'où les oscillations parasites apparaissant toujours dans les solutions principales.

Ce phénomène très bien connu des spectroscopistes (surtout de Fourier) donne naissance à des pieds négatifs. En effet même si l'entrée $e(x)$ était positive, sa convolution par la fonction sinc donne des parties négatives.

D'où le nom d'apodisation donné aux méthodes qui après ce filtrage inverse (qui fournit un filtre de gain 1 sur toute la bande passante instrumentale) pondèrent par une fonction $P(v)$ dite fonction d'apodisation.

Le choix de $P(v)$ ne peut se faire que selon un critère donné (remarquons que si l'on n'a aucune connaissance de l'objet mieux vaut ne rien faire : ni filtrage inverse, $n i$ apodisation. L'estimation de $e(x)$ par la sortie $s(x)$ est aussi bonne que toute autre !). Arsac [2], Jacquinot et al. [14] par exemple ont proposé des formes de $P(v)$ adaptées à des cas particuliers (satellite dans l'aile d'une raie, meilleur rendu du contraste, des dérivées $1^{\text {re }}$ ou $2^{\mathrm{e}}$, etc...) Frieden [15] a proposé une pupille superrésolvante dont la diracité est très grande mais qui présente des lobes parasites de grande amplitude. La pupille utilisée est formée d'une somme de Prolate Spheroidal Wave Functions [16].

Les inconvénients de cette méthode :

- gain faible en pouvoir séparateur,

- oscillations résiduelles,

- l'apodisation améliore certaine caractéristique de l'estimée mais le fait au prix d'une perte de qualité par ailleurs.

Frieden [8] a montré que le rapport $v_{\mathrm{utile}} / v_{\mathrm{co}}$ est directement lié au signal/bruit dans l'image. Quand $S / b=1, v_{\mathrm{utile}}=0$ et il est inutile d'opérer un filtrage inverse.

5.3.2 La méthode de Van-Cittert [17]. - Cette méthode opère dans l'espace des $x$. C'est une méthode itérative de filtrage inverse. Bracewell, R. S. and Roberts, J. A. [18], Jansson, L. A. [19] et Coupinot, G. [20] ont étudié et utilisé cette méthode. Le schéma est le suivant :

étape $k$ :

$$
\begin{aligned}
\widehat{s_{k}}(x) & =\widehat{e}_{k}(x) * A(x) \\
\widehat{e_{k+1}}(x) & =\widehat{e_{k}}(x)+\left[s(x)-\widehat{s_{k}}(x)\right]
\end{aligned}
$$

avec

$$
\hat{e}_{0}(x)=s(x) \text {. }
$$

La méthode pour $k \rightarrow \infty$ tend vers le filtrage inverse. Elle ne converge que si $|1-a(v)|<1, \forall v$. Si $a(v) \rightarrow 0$, on voit que le bruit est amplifié linéairement à chaque itération. Nous verrons plus loin que cette méthode a été récemment améliorée.

5.3.3 Méthodes statistiques. - Helstrom, C. W. [21] utilisant une méthode statistique réussit à trouver un filtre réalisant une approximation de $\hat{e}(x)$ aux moindres carrés et une minimisation des oscillations dans $\hat{e}(x)$. Le filtre trouvé tient compte du rapport $S / b$ mais on doit par tâtonnements trouver le coefficient du compromis fidélité/régularité.

Si l'on accepte de perdre la régularité de la restitution $\hat{e}(x)$, Fellgett, P. B. et Linfoot, E. M. [22] ont montré que le filtre optimal (sens des moindres carrés) est lié à la quantité suivante :

$$
I(v)=\log \left[1+\frac{\Gamma_{e}(v)}{\Gamma_{n}(v)}|a(v)|^{2}\right]
$$

où $\Gamma_{e}$ et $\Gamma_{n}$ sont les $\mathrm{d}$. s. e. de l'entrée et du bruit chiffrant la qualité de l'information transmise par le filtre et observée par $s(x)$.

Le filtre $F(v)$ s'écrit alors :

$$
F(v)=\frac{1}{a(v)}\left[1-\mathrm{e}^{-I(v)}\right]
$$

Le terme $1 / a(v)$ traduit le filtrage inverse qui n'est possible que si $I \rightarrow \infty$. On montre aussi que l'erreur

$$
\varepsilon^{2}=\int_{-\infty}^{+\infty}|e(x)-\hat{e}(x)|^{2} \mathrm{~d} x
$$

décroît exponentiellement avec $I$.

Dans la même approche la philosophie de Backus, G. et Gilbert, F. [23] est de trouver un filtre $F(v)$ qui minimise séparément

- la puissance moyenne du bruit de sortie : $Q_{1}^{2}$ et

$$
-\quad \int_{-\infty}^{+\infty} x^{2}[A(x) * f(x)]^{2} \mathrm{~d} x=Q_{2}^{2} \text {. }
$$

Cette dernière quantité mesure la diracité. Si l'on minimise la combinaison linéaire

$$
\alpha Q_{1}^{2}+\beta Q_{2}^{2}
$$

on peut trouver une relation entre la diracité $Q_{2}$ et la puissance de bruit acceptée à la sortie de $F(v)$. Mais il nous semble qu'en plus des inconvénients liés aux méthodes linéaires cette méthode fournit une mesure $Q_{2}$ du pouvoir de résolution assez arbitraire.

6. Possibilité d'extension de bande. - A l'opposé des méthodes de filtrage inverse agissant dans la bande passante $\left[-v_{\text {co }}, v_{\text {co }}\right]$ nous allons voir maintenant des méthodes qui tentent de retrouver $e(x)$ avec un pouvoir de résolution accru.

Il est évident que ces méthodes doivent s'appuyer sur une certaine connaissance a priori de $e(x)$. Nous 
insistons sur ce point. Nous verrons plus loin tout le parti que l'on peut tirer d'une information a priori.

6.1 MÉThOdes UTILISANT LA PROPRIÉTÉ $e(x) \equiv 0$, $\forall x \notin[-X, X]$. - Comme on l'a déjà vu cette propriété se traduit par l'analyticité de $E(v)=\operatorname{TF}(e(x))$.

Beaucoup d'auteurs ont essayé de tirer parti de cette information. On peut immédiatement penser à développer $E(v)$ en série de Taylor. Dans la pratique, et en présence d'un très faible bruit, ceci est rigoureusement impossible [24] Mac Phie, [25] Ville et [26] Wolter ont montré qu'il était nécessaire d'avoir un rapport $S / b \sim 1000$ pour pouvoir prolonger d'environ $10^{-2}$. Ceci est manifestement dû aux incertitudes sur les dérivées d'ordre croissant.

Une autre façon de traduire l'analyticité de $E(v)$ est utilisée par J. P. Scheidecker [27] qui essaye à l'aide des polynômes de Bernstein de prolonger $E(v)$. On peut atteindre dans un cas non bruité une fréquence d'extrapolation $v_{\mathrm{ex}} \sim 1,5 v_{\mathrm{co}}$ mais cette méthode est très sensible au bruit. Les polynômes divergent très vite au-delà de $v_{\text {ex }}$.

D'autres auteurs (Harris [28] et Robaux [29]) utilisent le fait qu'on peut appliquer la formule d'interpolation de Shannon à $E(v)$. Harris applique brutalement la formule et il obtient tous les inconvénients déjà rencontrés dans la résolution matricielle directe (oscillations...). Il présente des résultats non bruités. $M^{\text {me }}$ Robaux remplace la fonction d'appareil réelle par une fonction apodisée partout positive et plus étroite.

Enfin, plusieurs auteurs (Scheidecker [27], Frieden [30], Williams et Chang [31]) ont tenté d'utiliser les propriétés des Prolate Spheroidal Wave Functions. Ces fonctions $\Psi_{n}$ forment une base complète orthonormale aussi bien sur un support borné que sur ]$-\infty,+\infty[$. Il suffit donc de décomposer $E(v)$ sur cette base, de calculer les coefficients du développement sur $\left[-v_{\text {co }}, v_{\text {co }}\right]$. Ceux-ci permettent théoriquement la construction de $E(v)$ sur ] $-\infty,+\infty[$.

Les problèmes posés par cette méthode sont nombreux :

- l'amplification du bruit extrapolé étudié par Frieden [30], Robaux [29] et Williams et Chang. [31],

- oscillations (négatives),

- difficulté du calcul des $\Psi_{n}$. On peut les calculer comme solution d'une équation intégrale ou d'une équation différentielle.

6.2 MÉTHOdes S’APPUYANT SUR LA POSITIVITÉ. Ce sont les méthodes qui se sont révélées les meilleures jusqu'à ce jour.

Nous citons, pour mémoire, la méthode brutale préconisée par Hogbom (1969 et non publiée). Partant des données fournies par le filtre inverse, il en prend la transformée de Fourier, obtient la S. P. de Bracewell. Si elle présente des pieds négatifs, il met la $S$. $P$. à zéro partout où elle est négative. Prenant la TF de cette nouvelle estimée, il repasse dans l'espace des fréquences et normalise cette TF sur $S(v) / a(v)$. Puis il repasse dans l'espace direct. Cette entrée estimée peut présenter encore des pieds négatifs. On les supprime encore, etc... Ce procédé force donc la convergence vers une solution positive mais sans extension de bande. Il se pose aussi dans cette méthode des problèmes de conservation de l'énergie. Nous ne connaissons pas les effets du bruit dans cette méthode.

Nous envisagerons ensuite deux méthodes de déconvolution positive.

Schell [33] utilise un algorithme itératif. On cherche une fonction $P(v)$ telle que son carré de convolution $P(v) * P(v)$ approche $S(v) / a(v)$. On a la récurrence :

$$
\begin{aligned}
P_{k+i}\left(v_{i}\right) & =\mathrm{Cte} \cdot \times \\
& \times \sum_{j=-(N+i) / 2}^{(N-i) / 2}\left[\frac{S\left(v_{j}\right)}{a\left(v_{j}\right)}-\left.P(v)^{* 2}\right|_{v=v_{j}}\right] \cdot P_{k}\left(v_{i+j}\right) .
\end{aligned}
$$

On peut ainsi élargir la bande au-delà des $2 N$ points connus sur $S\left(v_{j}\right) / a\left(v_{j}\right)$. Le problème qui se pose est de savoir dans quelle mesure extrapoler cette bande en fonction du $S / b$ dans la sortie. Nous verrons plus loin ce problème.

Y. Biraud [34], [35] a mis au point une méthode itérative qui est la suivante. La seule donnée d'entrée est le quotient $S(v) / a(v)$ sur le support $\left[-v_{u}, v_{u}\right]$ soit $H(v)$. La méthode tend à approximer $H(v)$ par une fonction $G(v)$ qui soit un carré de convolution

$$
G_{n}(v)=F_{n}(v) * F_{n}(v)=F_{n}(v)^{* 2} .
$$

L'approximation se fait évidemment sur $\left[-v_{u}, v_{u}\right]$ et au sens des moindres carrés. On minimise donc

$$
P_{n}=\int_{-v_{u}}^{+v_{u}}\left|H(v)-G_{n}(v)\right|^{2} \mathrm{~d} v .
$$

Cette quantité $P_{n}$ est comparée, à chaque étape de l'itération, à la puissance moyenne de bruit sur la bande utile

$$
Q=\int_{-v_{u}}^{+v_{u}}|n(v)|^{2} \mathrm{~d} v .
$$

L'algorithme se déroule en ordinateur sur des fonctions échantillonnées (au pas de Shannon) et procède comme suit :

Etape no 1 :

$$
F_{1}(v) \text { arbitraire de support }\left[-v_{u} / 2, v_{u} / 2\right]
$$

$G_{1}(v)=F_{1}(v)^{* 2}$ est donc de support $\left[-v_{u}, v_{u}\right]$.

Etape no 2 :

$$
F_{2}(v)=F_{1}(v)+\varpi_{1}(u, v, w, l)
$$

$\varpi_{1}(u, v, w)$ est une fonction perturbatrice n'agissant sur $F_{1}(v)$ qu'aux points d'échantillonnage $F(0)$, $F( \pm l . h)$. Voir figure 8. Alors

$$
G_{2}(v)=F_{1}(v)^{* 2}+2 F_{1}(v) * \varpi_{1}(v)+\varpi_{1}^{* 2}(v)
$$




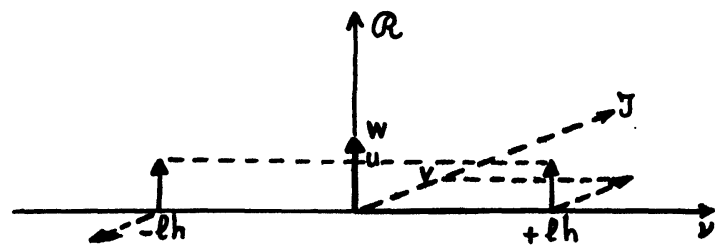

Fig. 8. - La perturbation dans la méthode de Biraud $\bar{\omega}(u, v, w, l)=w \delta(v)+u[\delta(v+l h)+\delta(v-l h)]-$

$$
-j v[\delta(v+l h)-\delta(v-l h)]
$$

est définie sur $\left[-v_{u}, v_{u}\right]$ et diffère de $G_{1}(v)$ en tout point. On calcule

$$
R_{2}(u, v, w)=\int_{-v_{u}}^{v_{u}}\left|H(v)-\left[G_{2}(v)-\varpi_{1}^{* 2}(v)\right]\right|^{2} \mathrm{~d} v
$$

et l'on cherche le triplet $u, v, w$ qui

i) minimise $P_{2}$,

ii) assure la conservation de l'énergie

$$
<G_{n}(v), \delta(v)>=H(0) .
$$

Cette deuxième condition est fondamentale.

Le calcul de $u, v, w$ qui pourrait se faire à l'aide de la méthode des multiplicateurs de Lagrange s'avère trop long en ordinateur. On se contente donc de dériver $\mathrm{P}_{2}$ par rapport à $u$ et $v$. D'où un système d'équation linéaire en $u$ et $v$ dont les solutions, fonction de $w$, sont reportées dans l'éq. (12) quadratique en $u, v$ et $w$. La perturbation ainsi calculée est ajoutée à $F_{1}$ et on calcule $P_{2}$.

La troisième étape calculera $\varpi_{2}(u, v, w, l)$ avec $l=2$.

La quatrième étape calculera $\varpi_{3}(u, v, w, l)$ avec $l=3$.

La $N$-ième étape calculera $\varpi_{N-1}(u, v, w, l)$ avec $l=N-1$ tel que

$$
(l-1) h=v_{u} / 2
$$

de telle sorte que $G_{N}(v)$ soit ıoujours définie sur $\left[-v_{u}, v_{u}\right]$.

Ensuite, on recommence le cycle $l=2,3, \ldots, N-1$, etc...

On trace la courbe d'erreur d'approximation $P_{n}=f(n)$.

On constate alors que :

i) après un certain nombre de cycles, $P_{n}$ ne diminue plus (Fig. 9),

ii) on est obligé d'étendre la bande passante spatiale. Ceci se fait en choisissant $l=N$,

iii) après addition d'un point supplémentaire sur $\mathrm{F}$ l'erreur se met à décroître immédiatement. Puis, le même phénomène de saturation de l'approximation apparaît après un certain nombre de cycles $l=2,3, \ldots, N$.

On rajoute alors encore un point supplémentaire $l=N+1$, etc... jusqu'à ce que l'erreur d'approximation $P_{n}$ soit comparable à la puissance moyenne de bruit.

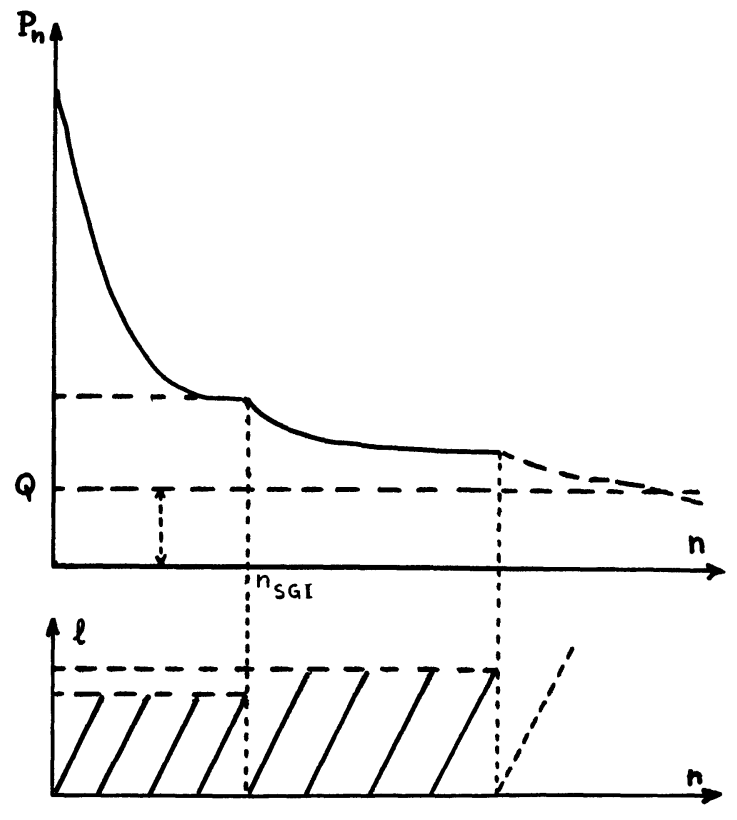

Fig. 9. - Variation de l'erreur d'approximation $\boldsymbol{P}_{\boldsymbol{n}}$ en fonction du numéro $n$ de l'itération. $1<n<n_{\mathrm{SGI}}$ : approximation de $S(v) / a(v)$ sur $\left[-v_{u}, v_{u}\right], n_{\mathrm{SGI}}<n$ : extension de la bande passante.

Le processus traduit ainsi la limitation due au bruit. Mais le fait qu'il utilise la connaissance $a$ priori de la positivité de l'entrée lui confère une insensibilité très grande au bruit. Des essais probants ont été obtenus avec des $S / b$ voisins de 1 .

Les figures 10 à 13 présentent quelques résultats obtenus par cette méthode.

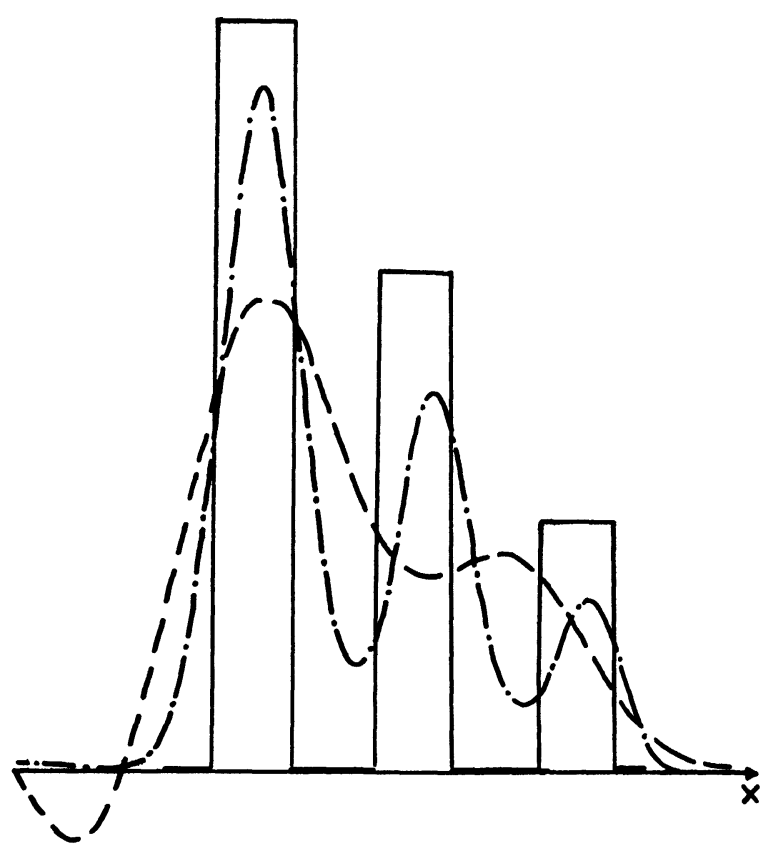

FIG. 10. - Exemple de déconvolution positive (Biraud) $S / b \infty$. L'entrée $s(x)$. - - La Solution Principale de Bracewell. - - La restitution positive. Cet exemple reprend celui de la figure 6. 


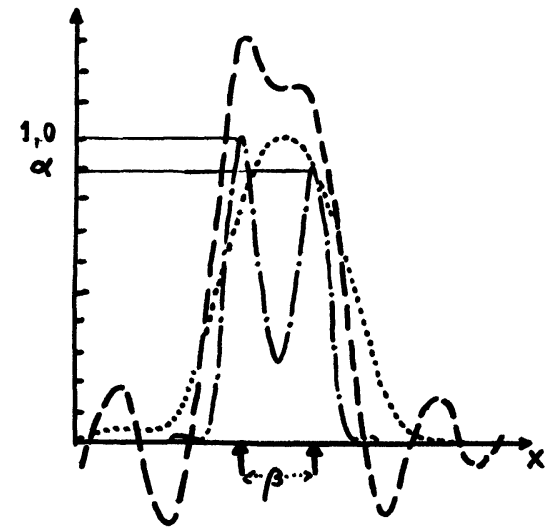

FIG. 11. - Application à la Radioastronomie : distribution de brillance E-W de Cygnus A. - - - - s(x). C'est la sortie brute du radiotélescope de Nançay travaillant à la longueur d'onde $11 \mathrm{~cm}$. - - La Solution Principale. -. - La restitution positive. Rapport $S / b \simeq 80$. Le pouvoir séparateur a été multiplié par 2,75. Les paramètres $\alpha$ et $\beta$ caractérisant les deux sources sont corrects à $1 \%$ près (comparaison avec des mesures à plus haute résolution).

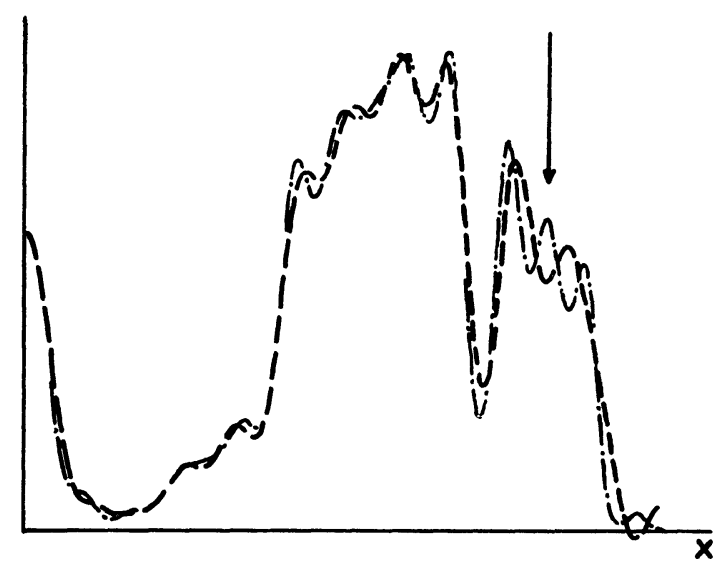

Fig. 12. - Application à l'Astronomie Optique. D'après Coupinot : distribution de brillance de l'anneau de Saturne. - - La sortie du filtre inverse. - - - La restitution positive. Celle-ci est plus contrastée et fait apparaître un motif nouveau dans la partie extérieure de l'anneau (marqué par la flèche).

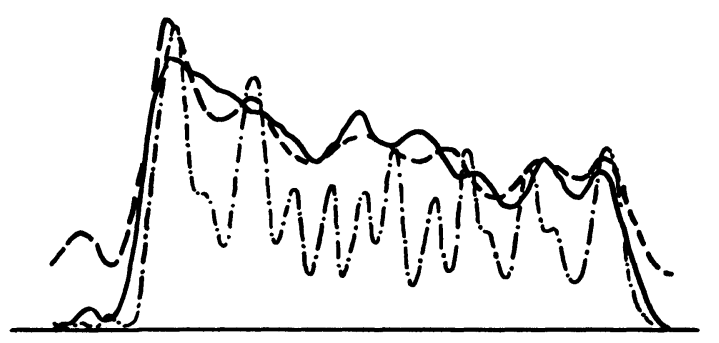

FIG. 13. - Application à la spectroscopie. D'après Botineau. Le spectre obtenu avec un spectomètre à grille de Girard. - - La Solution Principale. - - - La restitution positive. Les raies mises en évidence correspondent effectivement à des raies connues.

6.3 MÉTHOdE TRADUISANT L'EXISTENCE D'UNE BORNE INFÉRIEURE ET D'UNE BORNE SUPÉRIEURE DANS $e(x)$ : Jansson et al. [36]. - Cette méthode est une méthode de van-Cittert améliorée.
Le schéma est le suivant :

$$
\begin{aligned}
\hat{s}_{k}(x) & =\hat{e}_{k}(x) * A(x) \\
\hat{e}_{k+1}(x) & =\hat{e}_{k}(x)+r(x)\left[s(x)-\widehat{s}_{k}(x)\right]
\end{aligned}
$$

avec les mêmes conditions initiales $\hat{e}_{0}(x)=s(x)$.

On voit apparaître la fonction de pondération $r(x)$

$$
r(x)=\operatorname{Cte} \cdot\left[1-2\left|\hat{e}_{k}(x)-0,5\right|\right]
$$

qui tend vers 0 si $\hat{e}_{k}(x)$ tend vers 0 ou 1 . On voit donc que les amplitudes $\hat{e}(x)$ seront contraintes à rester dans la bande $[0,1]$.

Ceci peut se généraliser au cas $C_{1}<\widehat{e}(x)<C_{2}$ ou même

$$
C_{1}(x)<\hat{e}(x)<C_{2}(x)
$$

Les résultats fournis par cette méthode présentent une résolution accrue et semblent peu sensibles aux incertitudes sur $A(x)$ (voir Fig. 14). Les questions sans réponse sont les influences de la cadence d'échantillonnage et du rapport $S / b$.

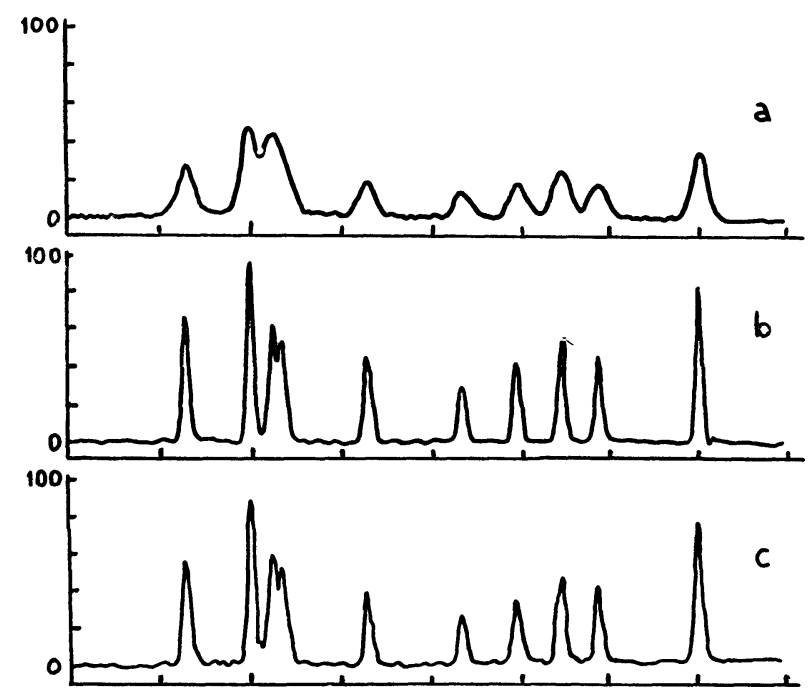

FIG. 14. - D'après Jansson et al. Méthode de van Cittert. Restitution contrainte aux bornes 0 et $100 \%$. a) Le spectre mesuré. b) La restitution en déconvolant par la réponse instrumentale mesurée $A(x)$. c) La restitution en déconvolant par une gaussienne approximant $A(x)$. Noter le peu de sensibilité de la méthode à l'incertitude sur la réponse instrumentale.

6.4 LES MÉTHODES DITES A MAXIMUM D'ENTROPIE. Il y a une analogie flagrante (Frieden [37] et J. G. Ables [38]) entre notre problème et la détermination d'une densité de probabilité $p(x)$ connaissant $n$ de ses moments :

$$
q_{n}=\int x^{n} p(x) \mathrm{d} x
$$

où $p(x)$ doit bien entendu être positif. Jaynes a montré 
que la solution la plus probable ne violant pas les données $q_{n}$ est celle qui a l'entropie maximale

$$
E=-\int p(x) \log p(x) \mathrm{d} x
$$

dont la solution est

$$
\widehat{p}(x)=\exp \left(-\sum_{i=1}^{N} \lambda_{i} x^{i}\right)
$$

où les $\lambda_{i}$ sont calculées à partir des moments $q_{n}$. Cette solution est évidemment non négative.

Ceci est la philosophie générale. Ensuite, deux approches ont été tentées.
Celle de Burg et Ables [38] qui maximisent l'intégrale

$$
I=-\int \log \hat{e}(x) \mathrm{d} x
$$

tout en minimisant l'écart entre la TF de $\widehat{e}(x)$ et $S(v) / a(v)=H(v)$.

Une méthode de multiplicateurs de Lagrange fournit la solution :

$$
\begin{array}{r}
\hat{e}(x)=\left(\mathcal{M}_{N+1} / 2 X\right) / \\
\qquad\left|1+\sum_{n=1}^{N} \mathcal{M}_{n} \exp \left(-j n \frac{\pi}{2 X}(x+X)^{2}\right)\right|
\end{array}
$$

où les $\mathcal{M}_{n}$ sont solutions de l'équation matricielle

$$
\left(\begin{array}{ccc}
H(0) & H\left(v_{1}\right) & H\left(v_{2}\right) \ldots H\left(v_{N}\right) \\
H\left(v_{1}\right) & H(0) & H\left(v_{1}\right) \ldots H\left(v_{N-1}\right) \\
\vdots & & \\
H\left(v_{N}\right) & \ldots \ldots \ldots \ldots & \ldots(0)
\end{array}\right)\left(\begin{array}{c}
1 \\
\mathcal{M}_{1} \\
\vdots \\
\mathcal{M}_{N}
\end{array}\right)=\left(\begin{array}{c}
\mathcal{M}_{N+1} \\
0 \\
\vdots \\
0
\end{array}\right)
$$

Le problème est encore celui d'une inversion de matrice qui dans les cas pratiques est entachée de bruit. A très fort $S / b$ la méthode a donné de bons résultats en Radioastronomie [38]. Mais Lacoss [39] a montré qu'elle était très sensible au bruit (dégradation très importante des résultats quand on passe d'un $S / b$ de 30 à 20). Il y a exagération des pics au-delà des vraies valeurs. Par contre l'énergie contenue dans une raie par exemple est bien restituée.

La méthode de Frieden considère l'énergie globale $E_{\text {Tot }}$ contenue dans l'image qu'il découpe en cellules élémentaires. Il définit la probabilité de présence d'un grain de lumière dans la case $x_{n}$ de $e(x)$ par

$$
p_{n}=\frac{e\left(x_{n}\right)}{E_{\text {Tot }}}
$$

il cherche à maximiser

$$
E=-\sum \hat{e}\left(x_{n}\right) \log \hat{e}\left(x_{n}\right)
$$

- en tenant compte du bruit (additif) auquel il ajoute une constante positive permettant de définir

$$
\sum n\left(x_{j}\right) \log n\left(x_{j}\right)
$$

- en tenant compte des points de mesure sur $S(x)$ et de la conservation de l'énergie totale. Il trouve alors une solution

$$
\hat{e}\left(x_{n}\right)=\exp \left(-1-\hat{\lambda}_{N+1}-\sum_{k=1}^{N} \hat{\lambda}_{k} A\left(x_{k}-x_{n}\right)\right) .
$$

Pour calculer les $\lambda_{k}$ il utilise la méthode de Newton-Raphson.

L'algorithme est valide à 1 ou 2 dimensions (voir figure 15).
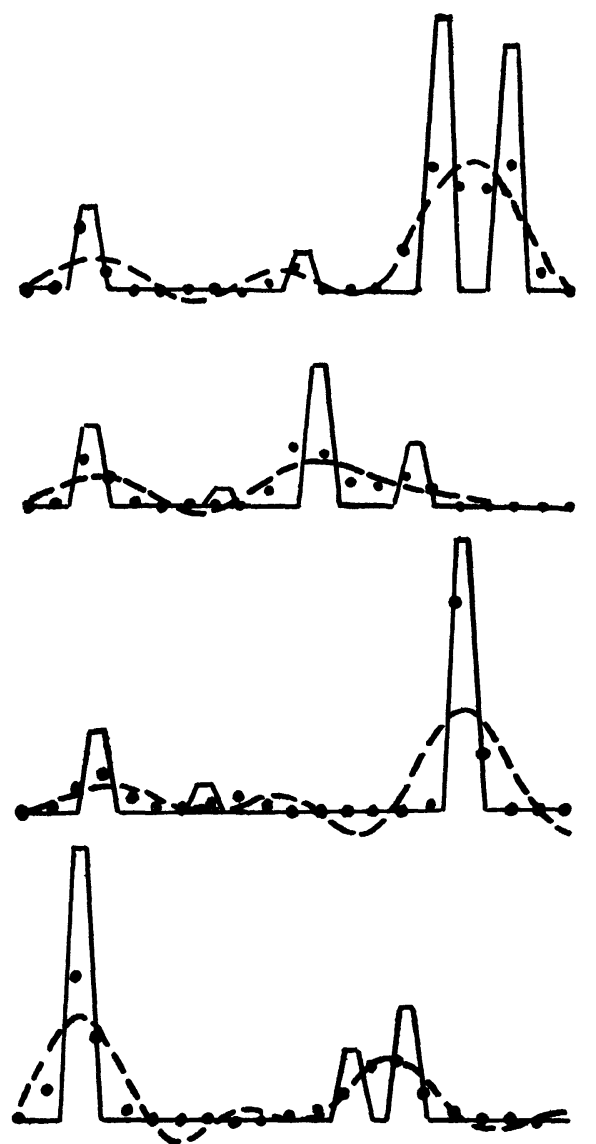

Fig. 15. - D'après Frieden. Méthode par maximum d'entropie. L'entrée $e(x)$ formée d'impulsions. - - La sortie du filtre inverse. $\cdots$ La restitution à entropie maximale. $S / b \simeq 20$

Néanmoins dans ces deux méthodes plusieurs points restent encore à étudier avec soin : le problème de 
l'échantillonnage (pas de Shannon ou différent ?) et celui de l'influence du bruit aussi bien en $S / b$ que vis-à-vis de la forme du spectre.

7. Conclusion. - Nous espérons avoir montré sur ces quelques exemples tous les problèmes qui se posent en déconvolution.

Nous pensons aussi :

i) que les connaissances a priori jouent un rôle fondamental, ii) que celles-ci sont très difficiles à traduire mathématiquement,

iii) que les méthodes non linéaires et contraintes se sont avérées très supérieures quant à la superrésolution.

Nous pensons enfin que des études approfondies tant théoriques que pratiques restent à faire.

Remerciements. - Nous tenons à remercier Bob Roy Frieden [8] pour l'aide considérable qu'il nous a apportée dans l'étude de nombreuses méthodes.

\section{Bibliographie}

[1] Biraud, Y. G., C. R. Colloque sur l'Impédance Mécanique, Lyon, mai 1972.

[2] ARsac, J., Transformation de Fourier et Théorie des Distributions (Dunod, Paris) 1961.

[3] Standish, C. J., IBM J. novembre 1967.

[4] VINOKUR, M., PIEEE 57, $\mathrm{n}^{\circ} 2$ (1969).

[5] Biraud, Y. G., C. R. Colloque sur l'Utilisation des Corrélateurs en Géophysique et Astronomie, Lyon, octobre 1972.

[6] Biraud, Y. G., Infrared Detection Techniques for Space Research. Manno and Ring Ed. (D. Reidel Pub. Co. Dordrecht) 1972, p. 313-31.

[7] Biraud, Y. G. et al., Etude Préliminaire de quelques problèmes relatifs à la Déconvolution. Rapport interne CEA-CENG 1974.

[8] Frieden, B. R., in Topics in Applied Physics, Vol. VI, Huang Ed. (Springer-Verlag, Heidelberg), à paraitre.

[9] Escudie, B., Thèse, Université de Lyon, 1967.

[10] Phillips, D. L., J. ACM 9 (1962) 84.

[11] Twomey, S., J. Franklin Inst. 279 (1965) 95.

[12] Tournarie, M., C. R. Hebd. Séan. Acad. Sci. 245 (1957) 1301.

[13] Dolby, R. M., Proc. Phys. Soc. 73 (1958) 81.

[14] Jacquinot, P. et Roizen-Dossier, B., Progr. Opt. 3 (1964) 31.

[15] Frieden, B. R., Appl. Opt. 9 (1970) 2489.

[16] Slepian, D. and Pollak, H. O., Bell. Syst. Tech. J. 40 (1961) 43.

[17] Van Cittert, P. H., Z. Phys. 69 (1931) 298.
[18] Bracewell, R. N. and Roberts, J. A., Aust. J. Phys. 7 (1954) 615-40.

[19] Jansson, P. A. et al., J. Opt. Soc. Am. 58 (1968) 1665.

[20] Coupinot, G., Thèse, Université P. et M. Curie, Paris, 1974.

[21] Helstrom, C. W., J. Opt. Soc. Am. 57 (1967) 297.

[22] Fellgett, P. B. and Linfoot, E. H., Phil. Trans. R. Soc. A 247 (1955) 369.

[23] Backus, G. and Gilbert, F., Phil. Trans. R. Soc. A 266 (1970) 123.

[24] Mac PhIE, R. H., Tech. Report, $\mathrm{n}^{\circ}$ 58, EERL, University of Illinois (Urbana, Ill.) 1962.

[25] Ville, J. A., Câbles et Transmissions (1956) 1-44.

[26] Wolter, H., Progr. Opt. 1 (1964) 202.

[27] Scheidecker, J.-P., Thèse de Spécialité, Paris, 1968.

[28] Harris, J. L., J. Opt. Soc. Am. 54 (1964) 7.

[29] Robaux, O., Thèse, Faculté d'Orsay, 1971.

[30] Frieden, B. R., J. Opt. Soc. Am. 57 (1967) 58.

[31] Williams, R. A. and Chang, W. S., J. Opt. Soc. Am. 56 (1966) 2.

[32] Barnes, C. W., J. Opt. Soc. Am. 56 (1966) 575.

[33] Schell, A. C., The Radio and Electronic Engineer 29 (1965) 21.

[34] Biraud, Y. G., C. R. $2^{\mathrm{e}}$ Colloque National GRETSI, Nice (1969).

[35] Biraud, Y. G., Astron. Astrophys. 1 (1969) 124.

[36] Jansson, P. A., J. Opt. Soc. Am. 60 (1970) 5.

[37] Frieden, B. R., J. Opt. Soc. Am. 62 (1972) 511.

[38] Ables, J. G., Astron. et Astrophys. Sup. Series, 15, 1974.

[39] LAcoss, R. T., Geophysics 36 (1971) 661. 